\title{
Unexplained Spectral Phenomena in the Interstellar Medium
}

\author{
Sun Kwok \\ Faculty of Science, The University of Hong Kong, Hong Kong, China \\ email: sunkwok@hku.hk
}

\begin{abstract}
We present an overview of the present observational status of unexplained spectral phenomena in the ISM. The possibility of organic molecules and solids as the carrier of the DIB, $217 \mathrm{~nm}$ feature, ERE, UIR, and the 21 and $30 \mu \mathrm{m}$ features is discussed.
\end{abstract}

Keywords. (ISM:) dust, extinction, (ISM:) planetary nebulae: general, ISM: lines and bands, ISM: molecules

The origin of diffuse interstellar bands (DIB), $217.5 \mathrm{~nm}$ feature, extended red emission (ERE), unidentified infrared emission (UIE) features, and the the 21 and $30 \mu \mathrm{m}$ features have remained unsolved after decades of efforts. Although the nature of the carrier is unknown, the strengths of the features suggest that their respective carriers are abundantly present in the ISM. These are also not isolated phenomena as they are observed in a wide range of celestial objects throughout the Universe. Could these phenomena be related to each other in any way? Is there a possibility that there exists a common carrier?

\section{Carriers of the spectral features}

Because of the strengths and ubiquitous nature of the features, the carrier must be made up of common, abundant elements. The element carbon is naturally assumed to play a major role. While the DIBs are widely accepted to be due to electronic transitions of gas-phase C-based molecules, the carrier of the $217 \mathrm{~nm}$ feature is more likely to be a carbonaceous solid such as amorphous carbon (Mennella et al. 1998), carbon onions (Iglesias-Groth 2004), hydrogenated fullerences (Cataldo \& Iglesias-Groth 2009), or polycrystalline graphite (Papoular \& Papoular 2009). Many proposals for the origin of the 21 $\mu \mathrm{m}$ feature have been proposed, including hydrogenated fullerenes (Webster 1995), SiC (Speck \& Hofmeister 2004), and thiourea groups attached to aromatic/aliphatic structures (Papoular 2011). Since ERE is the result of photoluminescience, the carrier is likely a semiconductor with a nonzero band gap and silicon nanoparticles have been suggested as a candidate (Ledoux et al. 1998, Witt et al. 1998). Other possibilities include QCC (Sakata et al. 1992), $\mathrm{C}_{60}$ (Webster 1993), and nanodiamonds (Chang et al. 2006).

In astronomical literature, PAH molecules are widely assumed to be the carrier of the UIE features. The PAH hypothesis states that the UIE features are the result of infrared fluorescence from small ( $\sim 50$ atoms) gas-phase PAH molecules being pumped by far-UV photons (Tielens 2008). The central argument for the PAH hypothesis is that single-photon excitation of PAH molecules can account for the $12 \mu \mathrm{m}$ excess emission observed in cirrus clouds in the diffuse ISM. However, the PAH hypothesis suffers from a number of problems: (i) PAH molecules have well-defined sharp features but the UIE features are broad; (ii) PAHs are primarily excited by UV, with little absorption in the visible but UIE features seen in PPN and reflection nebulae with no UV radiation; (iii) the predicted strong and narrow gas phase features in the UV are not seen in interstellar 
extinction curves; (iv) no PAH molecules have been detected in spite of the fact that the vibrational and rotational frequencies are well known; (v) no PAH emission spectrum has been able to reproduce the UIE spectrum w.r.t. either band positions or relative intensities (Cook et al. 1998); (vi) the shapes and peak wavelengths of the UIE features are independent of temperature of the exciting star, which raises doubts about radiative excitation mechanism; and (vii) in order to fit the astronomical observations, the PAH model has to appeal of a mixture of PAHs of different sizes, structures, and ionization states, as well as artificial broad intrinsic line profiles.

\section{Complex organics with disorganized structures}

In addition to the $s p^{2}$ (graphite and $\mathrm{PAH}$ ) and $s p^{3}$ (diamonds) structures, carbon compounds can also be in amorphous forms. Some carbonaceous compounds (HAC, coal, petroleum) are known to have a mixed $s p^{2} / s p^{3}$ structures with no well-defined geometric structural patterns. It has been suggested that mixed aromatic/aliphatic organic nanoparticles (MAON) may be responsible for the UIE features (Papoular 2001, Kwok \& Zhang 2011). Discussions on how such structures can contribute to our understanding of the ERE, $217 \mathrm{~nm}$, and the UIE features be found in the papers of Cataldo, Duley, Jones, Pino in these proceedings.

\section{Organics in the Solar System}

Although the traditional picture is that the Solar System is primarily made up of minerals, metals, and ices, organic compounds are now increasingly recognized as a common component in the Solar System. Complex organics have been found in meteorites, asteroids, comets, interplanetary dust particles, and planetary satellites. Although space scientists use different terms to refer to these organics (IOM in meteorites, tholins in Titan), any similarity in the chemical structures of these organics with the organics in the interstellar medium may suggest an interstellar origin of the Solar System organics.

\section{Acknowledgements}

This work is supported in part by a grant from the HKRGC (HKU 7027/11P).

\section{References}

Cataldo, F., \& Iglesias-Groth, S. 2009, MNRAS 400, 291

Chang, H.-C., Chen, K., \& Kwok, S. 2006, ApJ 639, L63

Cook, D. J., et al. 1998, JPCA 102, 1465

Iglesias-Groth, S. 2004, ApJ 608, L37

Kwok, S., Zhang, Y. 2011, Nature 479, 80

Ledoux, G., et al. 1998, A\&A 333, L39

Mennella, V., Colangeli, L., Bussoletti, E., Palumbo, P., \& Rotundi, A. 1998, ApJ 507, L177

Papoular, R. 2001, A\&SA 378, 597

Papoular, R. 2011, MNRAS 415, 494

Papoular, R. J., \& Papoular, R. 2009, MNRAS 394, 2175

Sakata, A., et al. 1992, ApJ 393, L83

Speck, A. K. \& Hofmeister, A. M. 2004, ApJ 600, 986

Tielens, A. G. G.. M. 2008, ARA\& A 46, 289

Webster, A. 1993, MNRAS 264, L1

Webster, A. 1995, MNRAS 277, 1555

Witt, A. N., Gordon, K. D., \& Furton, D. G. 1998, ApJ 501, L111 